\title{
VLT/UVES constraints on the cosmological variability of the fine-structure constant ${ }^{\star}$
}

\author{
S. A. Levshakov ${ }^{1}$, M. Centurión ${ }^{2}$, P. Molaro ${ }^{2}$, and S. D’Odorico ${ }^{3}$ \\ 1 Department of Theoretical Astrophysics, Ioffe Physico-Technical Institute, 194021 St. Petersburg, Russia \\ e-mail: lev@astro.ioffe.rssi.ru \\ 2 Osservatorio Astronomico di Trieste, via G. B. Tiepolo 11, 34131 Trieste, Italy \\ 3 European Southern Observatory, Karl-Schwarzschild-Strasse 2, 85748 Garching bei München, Germany
}

\section{Received 10 August 2004 / Accepted 12 January 2005}

\begin{abstract}
We propose a new methodology for probing the cosmological variability of $\alpha$ from pairs of Fe II lines (SIDAM, single ion differential $\alpha$ measurement) observed in individual exposures from a high resolution spectrograph. By this we avoid the influence of the spectral shifts due to $(i)$ ionization inhomogeneities in the absorbers; and (ii) non-zero offsets between different exposures. Applied to the Fe II lines of the metal absorption line system at $z_{\text {abs }}=1.839$ in the spectrum of Q 1101-264 obtained by means of the UV-Visual Echelle Spectrograph (UVES) at the ESO Very Large Telescope (VLT), SIDAM provides $\Delta \alpha / \alpha=$ $\left(2.4 \pm 3.8_{\text {stat }}\right) \times 10^{-6}$. The $z_{\text {abs }}=1.15$ Fe II system toward HE $0515-4414$ has been re-analyzed by this method thus obtaining for the combined sample $\Delta \alpha / \alpha=\left(0.4 \pm 1.5_{\text {stat }}\right) \times 10^{-6}$. These values are shifted with respect to the Keck/HIRES mean $\Delta \alpha / \alpha=$ $\left(-5.7 \pm 1.1_{\text {stat }}\right) \times 10^{-6}$ (Murphy et al. 2004) at very high confidence level $(95 \%)$. The fundamental photon noise limitation in the $\Delta \alpha / \alpha$ measurement with the VLT/UVES is discussed to figure the prospects for future observations. It is suggested that with a spectrograph of $\sim 10$ times the UVES dispersion coupled to a $100 \mathrm{~m}$ class telescope the present Oklo level $\left(\Delta \alpha / \alpha \geq 4.5 \times 10^{-8}\right)$ can be achieved along cosmological distances with differential measurements of $\Delta \alpha / \alpha$.
\end{abstract}

Key words. cosmology: observations - line: profiles - galaxies: quasars: absorption lines galaxies: quasars: individual: Q 1101-264, HE 0515-4414

\section{Introduction}

The variability of the fundamental physical constants over cosmic time, firstly proposed almost seven decades ago (Milne 1937; Dirac 1937), has been subsequently investigated in various aspects by many authors (for a review see, e.g., Uzan 2003).

The Sommerfeld fine-structure constant, $\alpha \equiv e^{2} / \hbar c$, which describes electromagnetic and optical properties of atoms, is the most suitable for time variation tests in both laboratory experiments with atomic clocks and astronomical observations. The value of this constant is known with high accuracy, $\alpha=1 / 137.03599976(50)$ (Mohr \& Tailor 2000), and its timedependence is restricted in the laboratory experiments at the level of $\mathrm{d} \ln [\alpha(t)] / \mathrm{d} t=\left(-0.9 \pm 2.9_{\text {stat }}\right) \times 10^{-15} \mathrm{yr}^{-1}$, corresponding to an upper limit of $|\mathrm{d} \ln [\alpha(t)] / \mathrm{d} t|<3.8 \times 10^{-15} \mathrm{yr}^{-1}$ (Fischer et al. 2004). At the cosmological time-scale $t \sim 10^{10} \mathrm{yr}$ $(z>1)$, this limit transforms into $|\Delta \alpha / \alpha| \equiv\left|\left(\alpha_{z}-\alpha\right) / \alpha\right|<$ $3.8 \times 10^{-5}$, if $\alpha_{z}$, the value of $\alpha$ at redshift $z$, is a linear function of $t$. The functional dependence of the gauge-coupling constants on $t$ is, however, unknown and theory predicts even

^ Based on observations performed at the VLT Kueyen telescope (ESO, Paranal, Chile). The data are retrieved from the ESO/ST-ECF Science Archive Facility. oscillations during the course of the cosmological evolution (e.g., Marciano 1984). In this regard the astronomical observations are the only way to test such predictions at different space-time coordinates (see, e.g., Mota \& Barrow 2004, where the effects of inhomogeneous space and time evolution of $\alpha$ are studied).

The astronomical measurements of the fine-structure splittings of emission lines in distant galaxies started by Savedoff (1956) are summarized in a recent comprehensive work by Bahcall et al. (2004, hereafter BSS): $\Delta \alpha / \alpha=\left(0.7 \pm 1.4_{\text {stat }}\right) \times$ $10^{-4}$ in the range $0.16<z<0.80^{1}$. The most stringent bound in the overlapping interval $z \leq 0.45$, stemming from the radioactive decay rates of certain long-lived nuclei found in meteoritic data, is set by Olive et al. (2004): $\Delta \alpha / \alpha=\left(-8 \pm 8_{\text {stat }}\right) \times 10^{-7}$. The time interval comparable with the meteoritic analysis is covered by the Oklo natural reactor $\left(\Delta t \sim 2 \times 10^{9} \mathrm{yr}\right)$. Recent reanalysis of the isotopic abundances in the samples taken from Oklo provides an intriguing result that the value of $\alpha$ was larger in the past: $\Delta \alpha / \alpha \geq 4.5 \times 10^{-8}$ (Lamoreaux \& Torgerson 2004).

At higher redshifts the variability of $\alpha$ can be tested by observations of small shifts between different ionic transitions

${ }^{1}$ Statistical errors, $\sigma_{\text {stat }} \equiv$ dispersion $/ \sqrt{n}$, are used in the text to specify the error of the sample mean, if not indicated otherwise. 
in the absorption-line spectra of quasars (Bahcall et al. 1967). This technique, now known as the alkali doublet (AD) method, was utilized in numerous studies (for a review, see BSS). The best constraint obtained by the $\mathrm{AD}$ method is $\Delta \alpha / \alpha=(-0.5 \pm$ $\left.1.5_{\text {stat }}\right) \times 10^{-5}$ (Murphy et al. 2001).

The AD method was generalized by Webb et al. (1999) and Dzuba et al. (1999, 2002) in the many-multiplet (MM) method which provides an order of magnitude improvement in the accuracy of the estimations of $\Delta \alpha / \alpha$. Being applied to 143 metal absorption systems (the dominant ions Mg II, Fe II at $z<1.8$, and Al II, Si II at $z>1.8$ ) identified in the Keck/HIRES spectra of quasars, the MM method indicates, opposite to the recent Oklo result, a decrease of $\alpha$ with cosmic time: $\Delta \alpha / \alpha=$ $\left(-5.7 \pm 1.1_{\text {stat }}\right) \times 10^{-6}$ in the redshift range $0.2<z<4.2$ (Murphy et al. 2004, hereafter MFWDPW).

A potential concern is that this result includes some systematics. Indeed, an increasing accuracy of the $\Delta \alpha / \alpha$ measurements requires a careful consideration of the intrinsic structure of the atomic transitions which is formed by the isotope shifts and hyperfine splittings (Levshakov 1994). For instance, the error of the mean $\sigma_{\langle\Delta \alpha / \alpha\rangle}=1.1 \times 10^{-6}$ corresponds to the error of the line center of $\sim 20 \mathrm{~m} \mathrm{~s}^{-1}$ (see Eq. (12) in Levshakov 2004, hereafter L04) which is about 40 times smaller than the isotope shift between ${ }^{26,24} \mathrm{Mg}$ II transitions $3 \mathrm{~s} \rightarrow 3 \mathrm{p}_{1 / 2}, 3 \mathrm{p}_{3 / 2}$, $\Delta v_{24-26} \simeq 850 \mathrm{~m} \mathrm{~s}^{-1}$ (Drullinger et al. 1980). Unfortunately, the influence of the isotope shifts cannot be well specified since we do not know the isotope abundances at different redshifts. If the isotope abundance ratio indeed varies with $z$, the isotope shifts may imitate the non-zero $\Delta \alpha / \alpha$ value (Ashenfelter et al. 2004; Kozlov et al. 2004, hereafter KKBDF). On the other hand, at metallicities of $Z \sim(0.1-1) Z_{\odot}$, - typical for the QSO systems with low ions, - the isotope abundances may not differ considerably from terrestrial (Murphy et al. 2003; Chand et al. 2004, hereafter CSPA).

The influence of unknown isotopic ratio and of another source of systematics caused by inhomogeneous ionization structure within the absorber can be considerably diminished if only one heavy element like, e.g., Fe II, is used in the $\Delta \alpha / \alpha$ measurement (L04). In spite of a rather low present accuracy of the theoretical calculations of the isotope shift parameters for atoms with more than one valence electron $(\sim 50 \%$, $\mathrm{KKBDF}$ ), the isotopic effect for Fe II (seven valence electrons in the configuration $3 d^{6} 4 s$ ) is less pronounced than that for $\mathrm{Mg}$ II (one valence electron in the configuration $3 \mathrm{~s}$ ) for two reasons: $(i)$ iron is heavier and its isotope structure is more compact; and ( $\mathrm{ii}$ ) the relative abundance of the leading isotope ${ }^{56} \mathrm{Fe}$ is higher ${ }^{2}$. Therefore systematic shifts in $\Delta \alpha / \alpha$ due to unknown isotopic compositions should be smaller in the analysis of the Fe II data.

This approach applied to the Fe II system identified at $z_{\mathrm{abs}}=1.15$ in the VLT/UVES spectrum of the bright quasar HE 0515-4414 $(B=15.0)$ gave $\Delta \alpha / \alpha=\left(-0.4 \pm 1.9_{\text {stat }} \pm\right.$ $\left.2.7_{\text {sys }}\right) \times 10^{-6}$ (Quast et al. 2004, hereafter QRL).

The most stringent limit on the variability of $\alpha, \Delta \alpha / \alpha=$ $\left(-0.6 \pm 0.6_{\text {stat }}\right) \times 10^{-6}$, standard deviation $\sigma_{\Delta \alpha / \alpha}=4 \times 10^{-6}$,

\footnotetext{
${ }^{2}$ The terrestrial isotope ratios are ${ }^{54} \mathrm{Fe}:{ }^{56} \mathrm{Fe}:{ }^{57} \mathrm{Fe}:{ }^{58} \mathrm{Fe}=$ 5.8:91.8:2.1:0.3, and ${ }^{24} \mathrm{Mg}:{ }^{25} \mathrm{Mg}:{ }^{26} \mathrm{Mg}=79: 10: 11$.
}

was claimed by CSPA. Their result is based on the VLT/UVES observations of 23 absorption systems $(0.4 \leq$ $z \leq 2.3)$ toward 18 QSOs. In this study, the MM method was applied to a more homogeneous ensemble of $\mathrm{Mg}$ II, Si II, and Fe II lines which are not strongly saturated and show a less complex structure as compared with the metal profiles from MFWDPW.

It should be noted, however, that the measurements with dispersions $\Delta \alpha / \alpha \sim 2 \times 10^{-6}\left(\Delta v \sim 60 \mathrm{~m} \mathrm{~s}^{-1}\right)$ are already at the sensitivity limit of the UVES and, hence, they may be affected by the data reduction procedure and/or by the changing in time characteristics of the device itself. There are three main sources of potential systematic errors in the absolute velocity scale: (i) temperature; and (ii) air pressure instability; and (iii) mechanical instabilities of unknown origin. For instance, a change of 1 millibar (or a change of $0.3{ }^{\circ} \mathrm{C}$ ) induces an error in radial velocities of $\sim 50 \mathrm{~m} \mathrm{~s}^{-1}$ (Kaufer et al. 2004).

In the present paper we investigate step by step the data reduction procedure usually applied to the VLT/UVES spectra in order to reveal possible systematics and to design the optimal measurement technique to avoid them. The analysis is based on another Fe II system identified in the absorption-line spectrum of quasar Q 1101-264.

The paper is organized as follows. In Sect. 2 we describe observations and data reduction performed to construct our Fe II sample. Small velocity shifts (equivalent to 0.1-0.2 pixel size) between the scientific exposures are analyzed in Sect. 3. Being ignored, such shifts may affect the shapes of the Fe II profiles in the co-added spectra, leading to their inconsistency. Section 4 presents the key procedure used to study the time dependence of $\alpha$, including error estimation. The results of our analysis are explained in Sect. 5. Section 6 gives the fundamental noise limitation in the $\Delta \alpha / \alpha$ measurement due to photon count and spectral profile. The quality factor similar to that introduced by Connes (1985) to optimize measurements of the stellar radial velocities is defined and calculated in this section. It can be used to control the sample dispersion in $\Delta \alpha / \alpha$. The obtained results and future prospects are discussed in Sect. 7. Our conclusions are given in Sect. 8 .

\section{Construction of the Fell sample}

The most accurate measurements of $\Delta \alpha / \alpha$ can be carried out with unsaturated Fe II lines which lie outside the Ly- $\alpha$ forest and are not corrupted by any other absorption and/or telluric lines. The components of the Fe II lines should be clearly distinguished. Besides, a QSO should be a bright object to provide a high $S / N$ ratio. These requirements are fulfilled for the $z_{\mathrm{abs}}=1.839$ system toward Q 1101-264 with $z_{\mathrm{em}}=2.145$ and $V=16.02$, which was discovered by Osmer \& Smith (1977). The absorption system was identified by Carswell et al. (1982) and investigated with high spectral resolution by Petitjean et al. (2000) and Dessauges-Zavadsky et al. (2003) who used the same UVES/VLT data as in the present work. This system was also studied with lower resolution in a series of works by Boksenberg \& Snijders (1981), Young et al. (1982), Carswell et al. (1984), and Lanzetta et al. (1987). 
Table 1. ESO UVES archive data on the quasar Q 1101-264.

\begin{tabular}{|c|c|c|c|c|c|c|c|c|c|c|}
\hline \multirow[b]{2}{*}{$\begin{array}{r}\text { Exp. } \\
\text { No. } \\
(1)\end{array}$} & \multirow[b]{2}{*}{$\begin{array}{l}\text { Setting } \\
\text { (2) }\end{array}$} & \multicolumn{5}{|c|}{ QSO } & \multicolumn{4}{|c|}{ ThAr } \\
\hline & & $\begin{array}{c}\text { Date, } \\
y-m-d \\
(3)\end{array}$ & $\begin{array}{c}\text { Time, } \\
\text { h:m:s } \\
(4)\end{array}$ & $\begin{array}{c}\text { Seeing, } \\
\operatorname{arcsec} \\
(5)\end{array}$ & $\begin{array}{c}T, \\
{ }^{\circ} \mathrm{C} \\
(6)\end{array}$ & $\begin{array}{c}P, \\
\mathrm{mb} \\
(7)\end{array}$ & $\begin{array}{c}\text { Date, } \\
\text { y-m-d } \\
(8)\end{array}$ & $\begin{array}{l}\text { Time, } \\
\text { h:m:s } \\
(9)\end{array}$ & $\begin{array}{c}\mathrm{T}, \\
{ }^{\circ} \mathrm{C} \\
(10)\end{array}$ & $\begin{array}{c}P, \\
\mathrm{mb} \\
(11)\end{array}$ \\
\hline 5 & 437 & $2000-02-11$ & 04:08:36 & $0.90 \pm 0.18$ & $11.2-11.3$ & $992.27-991.87$ & $2000-02-10$ & $19: 23: 55$ & $10.9-11.0$ & 990.34 \\
\hline 6 & 860 & 2000-02-11 & 04:09:47 & - & - & - & 2000-02-10 & $19: 25: 01$ & - & - \\
\hline 11 & 437 & 2000-02-12 & $03: 51: 57$ & $0.93 \pm 0.11$ & $11.4-11.4$ & 992.10-991.99 & 2000-02-12 & $13: 20: 28$ & $11.3-11.4$ & 993.07 \\
\hline 12 & 860 & 2000-02-12 & 03:53:11 & - & - & - & $2000-02-12$ & $13: 21: 44$ & - & - \\
\hline 19 & 437 & $2000-02-13$ & $02: 49: 57$ & $0.78 \pm 0.10$ & $11.4-11.5$ & 991.90-991.74 & & & & \\
\hline 20 & 860 & $2000-02-13$ & $02: 51: 14$ & - & - & - & & & & \\
\hline 21 & 437 & $2000-02-16$ & $03: 30: 15$ & $0.97 \pm 0.32$ & $11.2-11.3$ & 994.93-994.41 & $2000-02-16$ & $13: 52: 31$ & $11.1-11.3$ & 994.66 \\
\hline 22 & 860 & $2000-02-16$ & $03: 31: 37$ & - & - & - & $2000-02-16$ & $13: 53: 58$ & - & - \\
\hline 23 & 437 & $2000-02-16$ & $04: 30: 52$ & $0.74 \pm 0.01$ & $11.2-11.3$ & 994.41-994.07 & & & & \\
\hline 24 & 860 & $2000-02-16$ & $04: 32: 14$ & - & - & - & & & & \\
\hline
\end{tabular}

Columns 6, 7, and 10 list temperature and air pressure inside the spectrograph at the beginning and the end of the exposure.

\subsection{Observations and data reduction}

The observations were acquired with the UVES at the VLT $8.2 \mathrm{~m}$ telescope at Paranal, Chile, and the spectral data were retrieved from the ESO archive. The high resolution spectra of Q 1101-264 were obtained during the UVES Science Verification programme for the study of the Ly- $\alpha$ forest (Kim et al. 2002). The spectra used here were recorded with a dichroic filter which allows to work with the blue and red UVES arms simultaneously as with two independent spectrographs. The standard settings with central wavelengths at $\lambda 437 \mathrm{~nm}$ and $\lambda 860 \mathrm{~nm}$ were used for the blue and red arms, respectively. From the blue spectra we used only order 102, and from the red spectra - orders 90 and 83, where Fe II lines suitable for the $\Delta \alpha / \alpha$ measurement are observed. Details of the observations are presented in Table 1. Columns 1 and 2 give the exposure archive number and the setting used. Columns 3-7 list the QSO data, and Cols. 8-11 the data of the closest in time calibration thorium-argon (ThAr) lamp.

In this work we analyze five exposures (No. 5/6, 11/12, $19 / 20,21 / 22$, and 23/24) of $3600 \mathrm{~s}$ obtained over four nights in February 2000 with seeing as indicated in Col. 5 of Table 1 . The slit widths were both set at 0.8 arcsec and the CCDs were readout in $1 \times 2$ binned pixels (spatial $\times$ dispersion direction). The resulting spectral resolution as measured from the ThAr emission lines is of $F W H M \simeq 6.0 \mathrm{~km} \mathrm{~s}^{-1}$ in the blue $(\lambda \sim 4570 \AA)$, and of $\simeq 5.4 \mathrm{~km} \mathrm{~s}^{-1}$ in the red $(\lambda \sim 7380 \AA)$

We used the UVES pipeline (the routines implemented in MIDAS-ESO data reduction package for UVES data) to perform the bias correction, inter-order background subtraction, flat-fielding, correction of cosmic rays impacts, sky subtraction, extraction of the orders in the pixel space and wavelength calibration. A modified version of the routine "echred" of the context ECHELLE inside MIDAS was used to calibrate in wavelength the echelle spectra without rebinning. In this way we have the reduced spectra with their original pixel size in wavelength. This corresponds to a sampling of $50 \mathrm{~m} \AA$
( $3.3 \mathrm{~km} \mathrm{~s}^{-1} /$ pix $)$ for the blue and $55 \mathrm{~m} \AA\left(2.2 \mathrm{~km} \mathrm{~s}^{-1} / \mathrm{pix}\right)$ for the red. Typical rms of the wavelength calibration is less than onefiftieth of a pixel, or $\sigma_{\text {rms }} \lesssim 1 \mathrm{~m} \AA\left(\$ 60 \mathrm{~m} \mathrm{~s}^{-1}\right)$. The observed wavelength scale of each spectrum was transformed into vacuum, heliocentric wavelength scale (Edlén 1966). At first stage of our analysis we followed the standard procedure and added together the single extracted spectra using weights proportional to the square of their $S / N$. Before being added, the spectra were

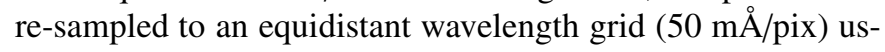
ing linear interpolation.

\subsection{A test for concordance of the Fe II profiles}

The Fe II profiles in the $z_{\mathrm{abs}}=1.8389$ systems toward Q 1101-264 consist of at least 6 subcomponents spread over $100 \mathrm{~km} \mathrm{~s}^{-1}$ (Dessauges-Zavadsky et al. 2003). The central component, seen in all but one Fe II lines from Table 2 (the undetected $\lambda 1611 \AA$ has the smallest oscillator strength), is marginally blended in the blue wing with weak component at $v=-17.6 \mathrm{~km} \mathrm{~s}^{-1}$ (see Fig. 1). Henceforth we will use this central component in the $\Delta \alpha / \alpha$ measurement.

We carefully checked that the profiles shown in Fig. 1 are free from cosmic rays and telluric absorptions. The combined spectra reveal high signal-to-noise ratios, $100 \leq S / N \leq 160$. The continuum levels are very well defined for all iron lines since in all cases there are large "continuum windows" at both sides of each Fe II absorption. But, nevertheless, we failed to find a model which could adequately describe the stacked profiles. Using the multicomponent Voigt profile fitting procedure we obtained the best normalized $\chi_{v}^{2}=3.995$ for the number of degrees of freedom $v=272$. The source of such high $\chi_{v}^{2}$ value is clearly seen in Fig. 1: some portions of the Fe II profiles are not in concordance with each other.

This inconsistency in the combined Fe II spectra may reflect some hidden problems in the standard data reduction procedure. We investigate this question in the next section. 

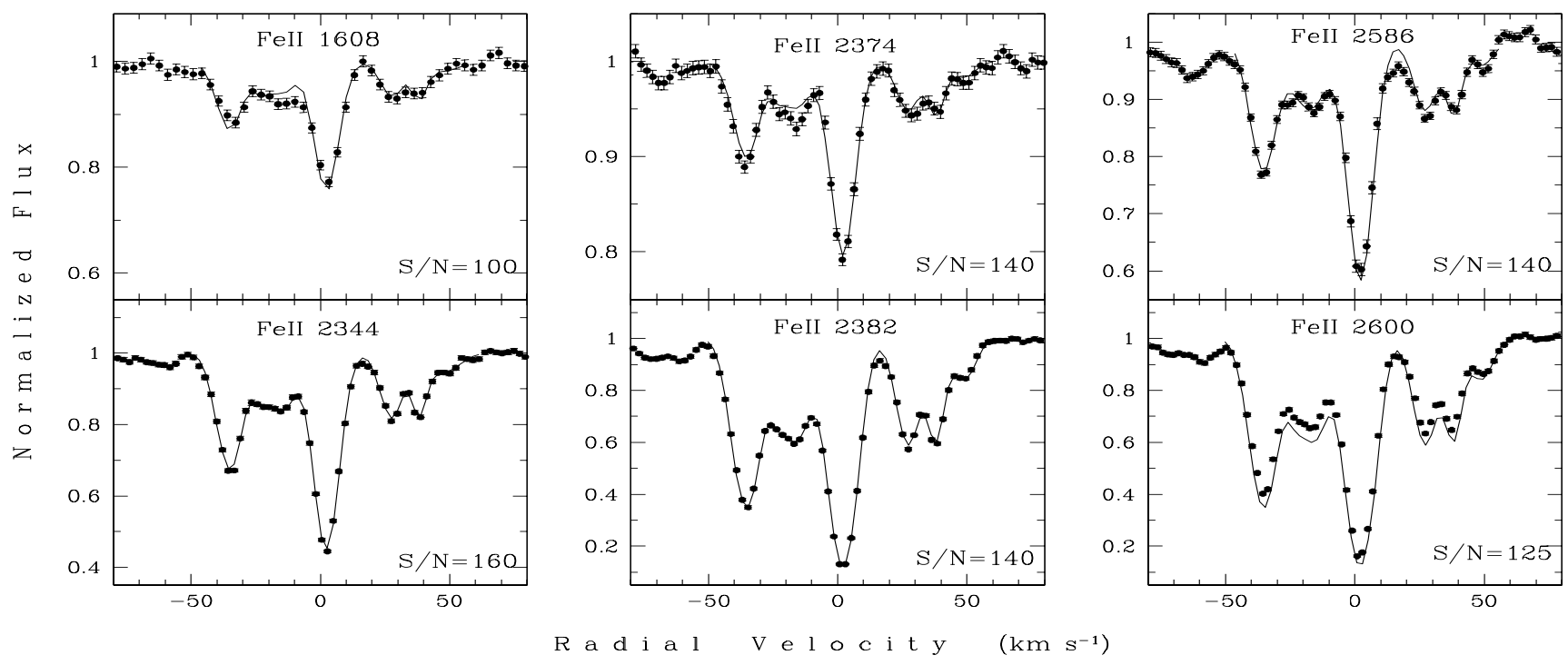

Fig. 1. Combined absorption-line spectra of Fe II associated with the $z_{\text {abs }}=1.839$ damped Ly- $\alpha$ system toward Q 1101-264 (normalized intensities are shown by dots with $1 \sigma$ error bars). The zero radial velocity is fixed at $z_{\text {abs }}=1.83888$. Smooth lines are the synthetic Voigt profiles convolved with the point-spread spectrograph function. The mean signal-to-noise ratio per pixel at the continuum level is indicated in each panel. The normalized $\chi_{\min }^{2}=3.995$ (the number of degrees of freedom $v=272$ ) shows that the Fe II profiles obtained in the framework of the standard data reduction procedure are not self-consistent.

Table 2. Atomic data of the Fe II transitions ${ }^{a}$, the sensitivity coefficients $Q^{b}$, and the isotope mass shift constants $k_{\mathrm{MS}}^{c}$. Estimated errors are given in parentheses.

\begin{tabular}{ccllr}
\hline \hline $\begin{array}{c}\text { Mlt. } \\
\text { No. }{ }^{d}\end{array}$ & $\begin{array}{c}\lambda_{\text {vac }}, \\
\AA\end{array}$ & $f$ & $Q$ & $\begin{array}{c}k_{\mathrm{MS}}, \\
\mathrm{cm}^{-1} \mathrm{amu}\end{array}$ \\
\hline $1 \mathrm{u}$ & $2600.1725(1)$ & 0.23878 & $0.035(4)$ & $-60(20)$ \\
$1 \mathrm{u}$ & $2586.6496(1)$ & 0.06918 & $0.039(4)$ & $-60(20)$ \\
$2 \mathrm{u}$ & $2382.7642(1)$ & 0.320 & $0.035(4)$ & $-63(20)$ \\
$2 \mathrm{u}$ & $2374.4603(1)$ & 0.0313 & $0.038(4)$ & $-63(20)$ \\
$3 \mathrm{u}$ & $2344.2130(1)$ & 0.114 & $0.028(4)$ & $-60(20)$ \\
$7 \mathrm{u}$ & $1611.20034(8)$ & 0.00136 & $0.018(5)$ & $-67(40)$ \\
$8 \mathrm{u}$ & $1608.45080(8)$ & 0.0580 & $-0.021(5)$ & $67(40)$ \\
\hline
\end{tabular}

${ }^{a}$ Based on the compilation of Murphy et al. (2003);

${ }^{b}$ Defined in Sect. $4 ;{ }^{c}$ Calculated by Kozlov et al. (2004);

${ }^{d}$ Multiplet numbers from Morton (2003).

\section{Velocity shifts between individual exposures}

In order to understand the origin of the discrepancy between Fe II profiles, we firstly investigated distortion and wavelength calibration. It turned out that two Fe II lines $\lambda 2344$ and $\lambda 2374$ lie close to the starting and end points of the echelle orders where distortions in the spectral sensitivity are the largest. These lines were excluded from the further analysis. The remaining Fe II lines are observed in the central parts of the echelle orders.

Then we selected isolated and unblended ThAr lines from these central spectral regions and compared their profiles. The time intervals between calibration exposures are large and the ThAr spectra were taken under different conditions. Namely, the differences between the mean temperatures and air pressures are $\Delta T_{5 / 6-11 / 12}=-0.40{ }^{\circ} \mathrm{C}, \Delta T_{11 / 12-21 / 22}=0.15{ }^{\circ} \mathrm{C}$, and $\Delta P_{5 / 6-11 / 12}=-2.73 \mathrm{mb}, \Delta P_{11 / 12-21 / 22}=1.59 \mathrm{mb}$, respectively (see Table 1 ). Changes in $T$ and $P$ lead to the velocity shifts between exposures. For instance, emission profiles plotted in Fig. 2 show that in the blue arm $\Delta \lambda_{5-11} \simeq-6 \mathrm{~m} \AA$ $\left(\Delta v \simeq-390 \mathrm{~m} \mathrm{~s}^{-1}\right)$ and $\Delta \lambda_{21-11} \simeq 4 \mathrm{m \AA}\left(\Delta v \simeq 260 \mathrm{~m} \mathrm{~s}^{-1}\right)$, whereas in the red arm $\Delta \lambda_{22-12}$ equals $\Delta \lambda_{21-11}$, as expected, but $\Delta \lambda_{6-12} \simeq-14 \mathrm{m \AA}\left(\Delta v \simeq-620 \mathrm{~m} \mathrm{~s}^{-1}\right)$ which is 1.6 times larger than $\Delta v_{5-11}$ (exposures 11 and 12 are taken for references). If the velocity drifts were entirely due to temperature and pressure changes, then we would observe a monotonous shift identical in the blue and red arms, as in the case of the $21 \mathrm{st}$ and 22nd exposures. The deviation from this behavior revealed for the 5th and 6th exposures indicates that the third factor some mechanical instabilities - may also be important. Since it is impossible to control what kind of distortions are caused by mechanical instabilities, we remove the scientific exposures 5 and 6 from the study.

This analysis shows that in order to avoid uncertainties in the wavelength calibration caused by changes in temperature and/or pressure, the calibration ThAr lamps must be taken just before and after the scientific exposure. If for some exposures the relative shifts of the ThAr lines in the blue and red arms turn out to be different, these exposures must be excluded from the consideration since they are influenced by mechanical instabilities which cannot be properly corrected.

Following the standard reduction procedure the remaining scientific exposures should be co-added to enhance $S / N$. However, this step can introduce additional uncertainties because every calibrated exposure has its own velocity offset. Note that the combination of the exposures by means of the MIDAS package requires the same starting wavelength, number of points, and the step size. Such procedure cancels out the original non-zero offsets between the individual exposures and smears out small shifts. This can perturb the line centroids, 

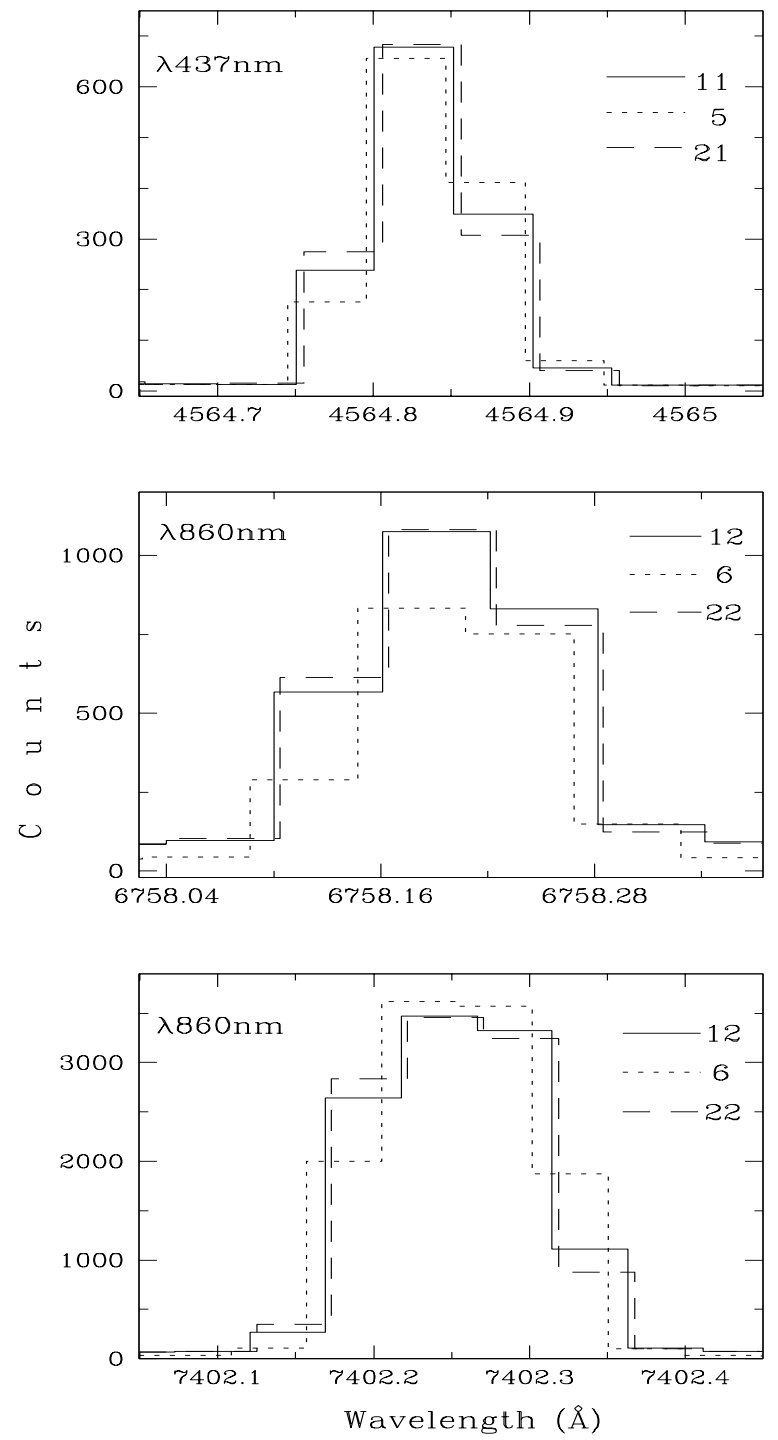

Fig. 2. Emission line profiles from the thorium-argon calibration lamp. The lines are placed close to the centers of the UVES echelle orders of different settings which are indicated at the top left hand corners in the panels. The wavelength step size corresponds to the original pixel width. The numbering of the histograms are in accord with the list in Table 1. The 11th and 12th exposures are taken as reference. Note the difference between the relative shifts $5-11$ and 6-12, while $21-11$ and 22-12 show identical velocity offsets.

especially those of narrow absorption lines with sharp intensity gradients. Since in the $\Delta \alpha / \alpha$ measurement the accuracy of the line position is the key factor, it is more appropriate to work with individual exposures which have lower $S / N$ rather than with a high $S / N$ combined spectrum.

Accounting for these arguments we selected the scientific exposures with Fe II lines shown in Fig. 3. All spectra are vacuum and heliocentric calibrated with only one ThAr spectrum (exposures 11 and 12 for the blue and red frames, respectively), they are not combined and not resampled. The unknown velocity offsets arising from such calibration are canceled out in the differential measurements of $\Delta \alpha / \alpha$ as described in the next section.
The key line in our approach is Fe II $\lambda 1608$ since its sensitivity coefficient is negative, while the other iron transitions have positive $Q$-values (Table 2). A relatively low strength of the $\lambda 1608$ line requires $S / N \gtrsim 30$ in individual exposures in order to measure its center accurately. This requirement is fulfilled for Q 1101-264 (see Fig. 4, where the $S / N$ values are indicated). It is also important that for this system the accuracy of the normalization of the Fe II profiles leaves no room for doubts since the local continua (the horizontal lines in Fig. 3) are calculated with high precision. In each panel in Fig. 3, dots with error bars indicate the mean intensities and their $1 \sigma$ uncertainties utilized in the linear regression analysis applied to determine the local continuum level. The uncertainty of the calculated continuum is found to be less than $1 \%$ for all selected Fe II spectra.

\section{The single ion differential $\alpha$ measurement}

The standard many-multilet (MM) technique (Webb et al. 1999; Dzuba et al. 1999, 2002) calculates $\Delta \alpha / \alpha$ using a quite complex procedure. This leads to potential systematic uncertainties which may affect both the line profile and the origin of the wavelength scale. The systematic effects are thoroughly discussed in Murphy et al. (2003), BSS, and L04. In this section we describe how to use a modified MM procedure to calculate $\Delta \alpha / \alpha$ directly from the differences between the wavelengths of a pair of Fe II transitions observed in the individual exposures. Thereafter this approach is called a "single ion differential $\alpha$ measurement" (SIDAM). An important point is that being applied to the lines from the same exposure, this method does not depend on the unknown offsets of the wavelength scale.

The MM method utilizes the fact that the energy of each line transitions depends individually on a change in $\alpha$. Namely, the frequency of each transition has its own relativistic correction to the changes in $\alpha$ which is expressed by the coefficient $q$ (Dzuba et al. 1999, 2002). Then, the systemic rest wavenumber $\omega_{z}=1 / \lambda_{z}$ is given by

$\omega_{z}=\omega_{0}+q(\Delta \alpha / \alpha)(2+\Delta \alpha / \alpha)$,

where $\omega_{0}=1 / \lambda_{0}$ is the laboratory wavenumber.

In fact, this approach is similar to the method developed by Varshalovich \& Levshakov (1993) in order to infer the cosmological variability of the proton-electron mass ratio, $\mu=$ $m_{\mathrm{p}} / m_{\mathrm{e}}$, from the analysis of molecular hydrogen $\mathrm{H}_{2}$ absorption lines. The dependence of the frequencies of electron-vibrorotational transitions on a change in $\mu$ differs for individual transitions and can be characterized by the dimensionless sensitivity coefficient $\mathcal{K}$. The estimation of $\Delta \mu / \mu$ can be obtained from linear regression analysis of the $\{z, \mathcal{K}\}$ pairs from a sample of the $\mathrm{H}_{2}$ lines (Potekhin et al. 1998; Levshakov et al. 2002b). Below this technique is applied to the $\Delta \alpha / \alpha$ measurement.

We can re-write (1) in linear approximation $(|\Delta \alpha / \alpha| \ll 1)$ in the form (L04):

$z_{i}=z_{0}+\kappa_{\alpha} Q_{i}$,

where $z_{i}=\lambda_{\mathrm{obs}, i} / \lambda_{0, i}-1, Q_{i}=q_{i} / \omega_{0, i}$ the dimensionless sensitivity coefficient, and the slope parameter $\kappa_{\alpha}$ is given by

$\kappa_{\alpha}=-2\left(1+z_{0}\right)(\Delta \alpha / \alpha)$. 

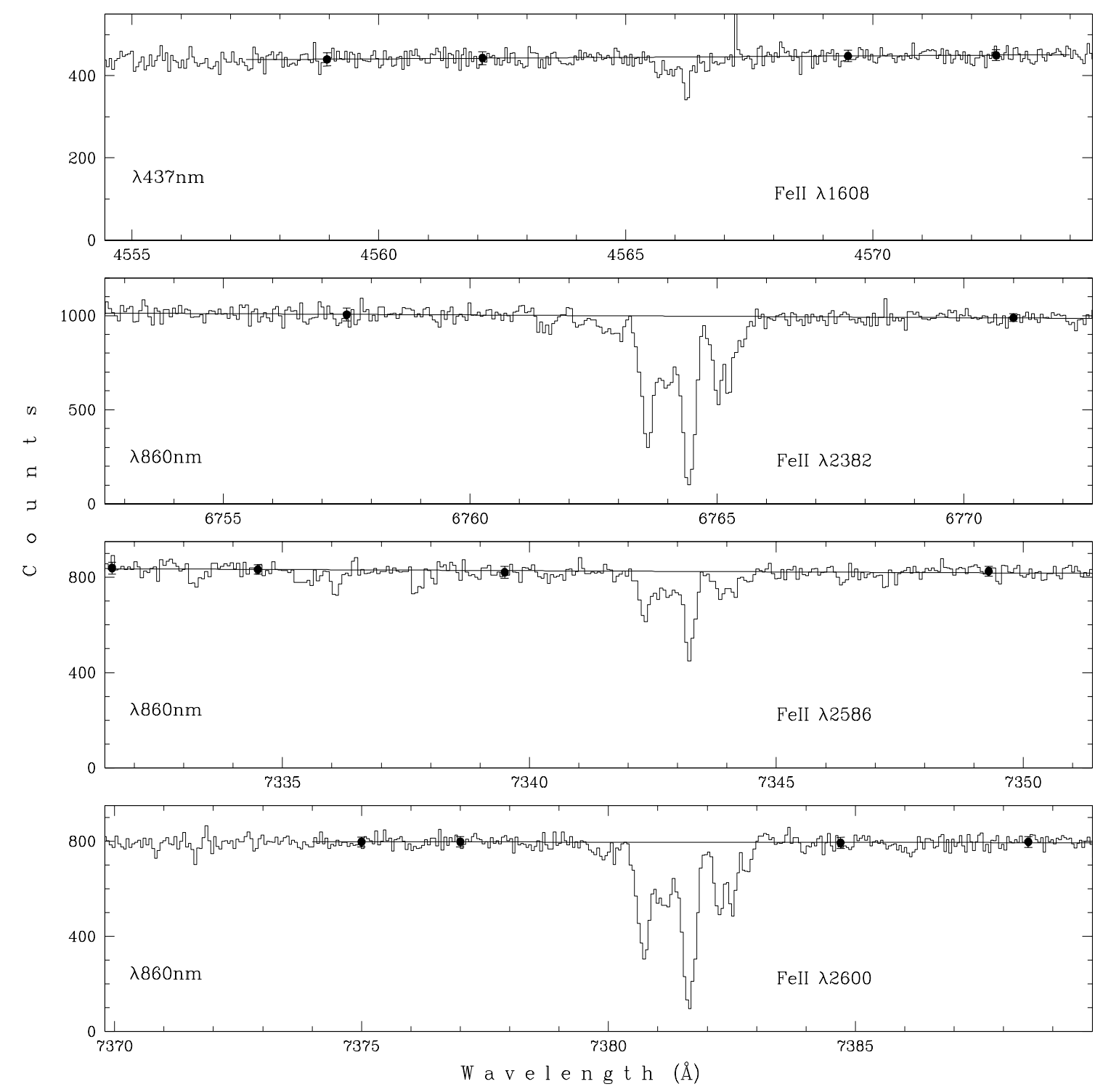

Fig. 3. Unnormalized portions of the Q 1101-264 spectra with the Fe II lines ( $\left.z_{\text {abs }}=1.839\right)$ selected for the $\Delta \alpha / \alpha$ measurement. All lines are located near the centers of the UVES echelle orders. The corresponding settings are indicated at the bottom left hand corners in each panel. Dots with error bars are the mean intensities and their $1 \sigma$ uncertainties - the values used to estimate the local continuum level by means of the linear regression analysis. For the selected Fe II lines, the continuum level is known with an accuracy better than $1 \%$.

If $\Delta \alpha / \alpha$ is non-zero, $z_{i}$ and $Q_{i}$ will be correlated. The slope $\kappa_{\alpha}$ and the intercept $z_{0}$ can be found from the linear regression analysis of the observed redshifts of the line centroids $z_{i}$ vs. $Q_{i}$ (for more details, see L04 and QRL, where this method is called a "regression" MM analysis, RMM).

In this approach, the value of $\Delta \alpha / \alpha$ can be directly estimated from a pair of lines with different sensitivity coefficients. It is easy to show that

$\frac{\Delta \alpha}{\alpha}=\frac{\left(z_{2}-z_{1}\right)}{\left(2+z_{1}+z_{2}\right)\left(Q_{1}-Q_{2}\right)}$,

or

$\frac{\Delta \alpha}{\alpha}=\frac{\left(z_{2}-z_{1}\right)}{2(1+\bar{z})\left(Q_{1}-Q_{2}\right)}$

The ratio $\left(z_{2}-z_{1}\right) /(1+\bar{z})$ is invariant and does not depend on systematic velocity shifts between different scientific exposures.
The computational procedure can be slightly modified if we use another variables similar to those introduced in the AD analysis by BSS.

Let us consider an absorption line system at redshift $z$ where a set of Fe II transitions is formed at the corresponding cosmic time $t$. Then, the ratio of the observed wavelengths $\lambda_{i}(t)$ to $(1+z)$ defines the rest wavelengths $\lambda_{i}^{\prime}(t)$ which may differ from their present-day values $\lambda_{i}(0)$, if $\Delta \alpha / \alpha \neq 0$. From (1) one obtains in linear approximation

$\lambda_{i}^{\prime}(t)=\lambda_{i}(0)\left(1-2 Q_{i} \Delta \alpha / \alpha\right)$.

Following BSS, we define $R(t)$ and $\eta$ by the relations

$R(t)=\frac{\lambda_{2}(t)-\lambda_{1}(t)}{\lambda_{1}(t)+\lambda_{2}(t)}$ 
and

$\eta=\frac{\lambda_{2}}{\lambda_{1}}-1$

The cosmological redshift of the absorber in the expression for $R$ cancels out and thus

$\frac{\lambda_{2}(t)-\lambda_{1}(t)}{\lambda_{1}(t)+\lambda_{2}(t)}=\frac{\lambda_{2}^{\prime}(t)-\lambda_{1}^{\prime}(t)}{\lambda_{1}^{\prime}(t)+\lambda_{2}^{\prime}(t)}$.

From Eqs. (6)-(9), one obtains after some algebra [taking into account $\eta(t) \approx \eta(0)]$

$\frac{\Delta \alpha}{\alpha}=\frac{1}{4\left(Q_{2}-Q_{1}\right)} \frac{\eta(\eta+2)}{\eta+1}\left(1-\frac{R(t)}{R(0)}\right)$.

In some cases (see Sect. 7.2) this formulation can be more convenient for calculations.

\subsection{Error estimations in the SIDAM technique}

In this section we calculate the uncertainty on individual $\Delta \alpha / \alpha$ measurement caused by the errors in the wavelengths. The standard method of error propagation is used.

From Eq. (4) we deduce (taking into account $z_{2} \approx z_{1}$ )

$\sigma_{\Delta \alpha / \alpha}^{2}=\frac{1}{4\left(Q_{1}-Q_{2}\right)^{2}}\left(\delta_{z_{1}}^{2}+\delta_{z_{2}}^{2}\right)$

where the relative error $\delta_{z}=\sigma_{z} /(1+z)$ is equal to

$\delta_{z}=\left(\delta_{\lambda}^{2}+\delta_{\lambda_{0}}^{2}\right)^{1 / 2}$.

If $\delta_{\lambda_{0}} \ll \delta_{\lambda}$ and $\delta_{\lambda_{1}} \approx \delta_{\lambda_{2}}$, then

$\sigma_{\Delta \alpha / \alpha}=\frac{1}{\sqrt{2}\left|Q_{1}-Q_{2}\right|} \delta_{\lambda}$

If the relative errors for both observational and laboratory lines are comparable, then the best estimation of $\Delta \alpha / \alpha$ is known with the error

$\sigma_{\Delta \alpha / \alpha}=\frac{1}{\left|Q_{1}-Q_{2}\right|} \delta_{\lambda}$

Using relation (14), it is easy to estimate the highest possible precision in the $\Delta \alpha / \alpha$ measurement with the laboratory Fe II data at hand. Assuming that the observed line centers are known with the accuracy $\delta_{\lambda} \simeq 5 \times 10^{-8}$ (see Table 2), one obtains $\tilde{\sigma}_{\Delta \alpha / \alpha} \simeq 8 \times 10^{-7}$. This corresponds to the uncertainty $\sigma_{\lambda}=0.25 \mathrm{~m} \AA\left(\Delta v=15 \mathrm{~m} \mathrm{~s}^{-1}\right)$ at $5000 \AA$. The value $\Delta v \sim 15 \mathrm{~m} \mathrm{~s}^{-1}$ is 4 times lower than the limit sensitivity of the UVES, and, hence, with good statistics (the sample size $\gtrsim 16$ ) $\Delta \alpha / \alpha$ can, in principle, be probed on the level of $\lesssim 10^{-6}$.

We note that the best precision reached today in the measurements of stellar radial velocities of relatively bright stars is a few $\mathrm{m} \mathrm{s}^{-1}$. Observations with HARPS provide, for example, the rms uncertainty of $2 \mathrm{~m} \mathrm{~s}^{-1}$ (Pepe et al. 2004) which is close to the HARPS fundamental noise limitation. For the $\Delta \alpha / \alpha$ measurement with UVES, the fundamental noise limitation due to spectral profile and photon count is considered in Sect. 6.

\subsection{Errors caused by the isotopic shifts}

The $\Delta \alpha / \alpha$ measurement on the scale of $\sim 8 \times 10^{-7}$ can be affected by the isotopic shifts if the ratio $\mathcal{I}={ }^{54} \mathrm{Fe} /{ }^{56} \mathrm{Fe}$ varies between the absorbers (the influence of the isotope ${ }^{57} \mathrm{Fe}$ is negligible because of its relatively low abundance). Following KKBDF and using their mass shift constants $k_{\mathrm{MS}}$ for Fe II transitions (Table 2), we can estimate the shift of the line center of gravity mimicking a non-zero $\Delta \alpha / \alpha$ :

$\delta \omega_{\mathrm{c}}=\frac{x}{100} \Delta \omega^{A^{\prime}, A} \approx \frac{x}{100} k_{\mathrm{MS}}\left(\frac{1}{A^{\prime}}-\frac{1}{A}\right)$,

where $A^{\prime}$ and $A$ are the isotope mass numbers, and $x$ (in per cents) shows the reduced fraction of the leading isotope $A$. If, for example, $x=10 \%$ (i.e. $\mathcal{I}_{z} / \mathcal{I}_{\odot} \simeq 3$ ), then $\delta \omega_{\mathrm{c}} \simeq$ $-0.004 \mathrm{~cm}^{-1}$ for Fe II transitions (see Table 1 in KKBDF).

Numerical simulations of the explosive yields show, however, that at low metallicities $Z<Z_{\odot}$ the ratio $\mathcal{I}_{z} / \mathcal{I}_{\odot} \lesssim 1$ (e.g., Chieffi \& Limongi 2004). Thus, in the high- $z$ absorbers one may expect an approximately constant isotopic shift $\delta \omega_{\mathrm{c}}=$ $-0.12 k_{\mathrm{MS}} / A^{2} \simeq 0.002 \mathrm{~cm}^{-1}$, which is equivalent to the positive shift $\Delta \alpha / \alpha \simeq 7.7 \times 10^{-7}$ (note that an offset of the velocity scale of $10-15 \mathrm{~m} \mathrm{~s}^{-1}$ can produce the same effect). However, this shift is canceled out in the differential $\Delta \alpha / \alpha$ measurements of a few high- $z$ absorbers having the same metallicities. This can essentially improve the limiting accuracy of $\sigma_{\Delta \alpha / \alpha} \sim 10^{-6}$ set by KKBDF for Fe II samples.

\section{Analysis and the $\Delta \alpha / \alpha$ results}

In this section we study the Fe II profiles selected from individual exposures and derive the position of the line centroid of the main absorption component seen at zero radial velocity in Fig. 4. The total number of the analyzed profiles is $L=16$. Since the line profiles are very complex, the distribution of the velocity components along the line of sight is crucial for the following $\Delta \alpha / \alpha$ measurement. To construct the model for the radial velocity distribution, we start with the analysis of the profiles from the individual scientific exposures to fix their reference frames - the mean $z_{\text {abs }}$ values. The following redshifts were determined: $z_{11 / 12}=1.8389040, z_{19 / 20}=1.8389041$, $z_{21 / 22}=1.8389065$, and $z_{23 / 24}=1.8389009$.

Our model is based on the natural assumption that Fe II lines have similar profiles, i.e., (1) the number of subcomponents $n_{\mathrm{s}}$ is identical for all Fe II lines; (2) the Doppler $b_{i}$ parameters are identical for the same $i$ th subcomponents; (3) the relative intensities of the subcomponents $r_{i, j}$; and (4) the relative radial velocity differences $\Delta v_{i, j}$ between the subcomponents are fixed for the absorber. We also assume that the main broadening is caused by bulk motion.

Then, the Fe II profile is described by the sum of $n_{\mathrm{s}}$ Voigt functions:

$\tau_{v}^{(\ell)}=N_{1} \sum_{i=1}^{n_{\mathrm{s}}} r_{i, 1} \mathcal{V}\left[\left(v-v_{\ell}-\Delta v_{i, 1}\right) / b_{i}\right]$,

where $\tau_{v}^{(\ell)}$ is the optical depth at radial velocity $v$ within the line $\ell, N_{1}$ is the column density of the main component, 


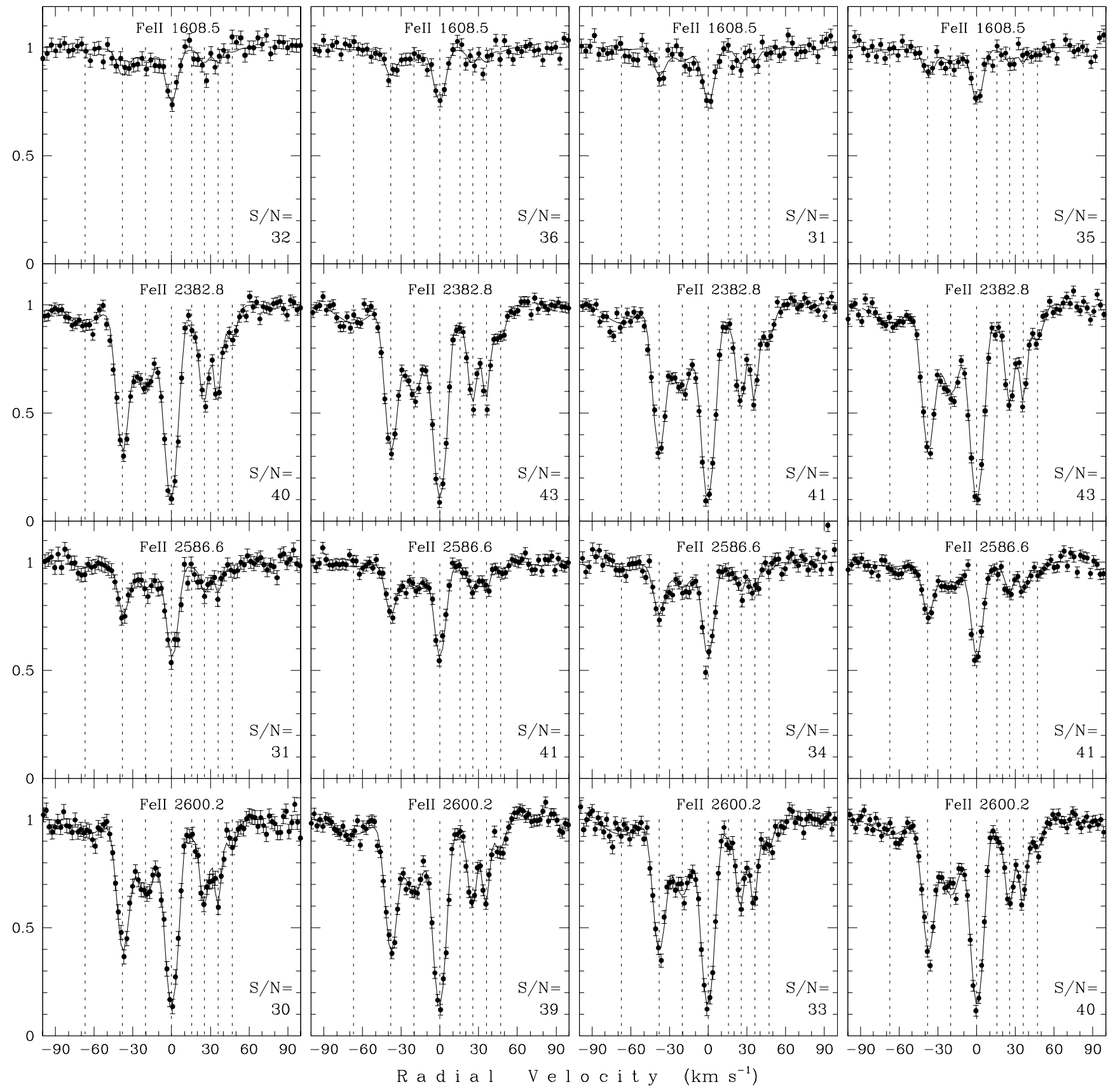

Fig. 4. Individual exposures of the Fe II lines in the spectrum of Q 1101-264 (normalized intensities are shown by dots with 1 $\sigma$ error bars) and over-potted synthetic profiles (smooth curves) calculated from the joint analysis. The minimization procedure gives $\chi_{\min }^{2}=1.097$ per degree of freedom (the number of data points $m=973$, the number of fitting parameters $p=39$ ). Dashed vertical lines mark positions of the individual components. The mean signal-to-noise ratio per pixel at the continuum level is indicated in each panel. Columns from the left to the right correspond to the exposures No. 11/12, 19/20, 21/22, and 23/24, in accord with Table 1. Spectral data shown in these columns are centered relative to the following fixed redshifts (from the left to the right): 1.8389040, 1.8389041, 1.8389065, and 1.8389009.

$r_{i, 1}=N_{i} / N_{1}, v_{\ell}$ is the center of the main component in the line $\ell$, and $\Delta v_{1,1}=0$.

Our model is fully defined by specifying $N_{1},\left\{b_{i}\right\}_{i=1}^{n_{\mathrm{s}}}$, $\left\{\Delta v_{i, 1}\right\}_{i=1}^{n_{\mathrm{s}}-1},\left\{r_{i, 1}\right\}_{i=1}^{n_{\mathrm{s}}-1}$, and $\left\{v_{\ell}\right\}_{\ell=1}^{L}$. All these parameters are components of the parameter vector $\theta=\left\{\theta_{1}, \theta_{2}, \ldots, \theta_{p}\right\}$. To estimate $\theta$ from the Fe II profiles, we minimize the objective function

$\chi^{2}(\theta)=\frac{1}{v} \sum_{\ell=1}^{L} \sum_{j=1}^{m_{\ell}}\left[\mathcal{F}_{\ell, j}^{\mathrm{cal}}(\theta)-\mathcal{F}_{\ell, j}^{\mathrm{obs}}\right]^{2} / \sigma_{\ell, j}^{2}$ where $\mathcal{F}_{\ell, j}^{\text {obs }}$ is the observed normalized intensity of the spectral line $\ell, \sigma_{\ell, j}$ is the experimental error within the $j$ th pixel of the line profile, and $v=\left(\sum_{\ell}^{L} m_{\ell}-p\right)$ is the number of degrees of freedom. $\mathcal{F}_{\ell, j}^{\mathrm{cal}}(\theta)$ is the calculated intensity convolved with the spectrograph point-spread function.

The synthetic profiles for the best $\chi^{2}$ are shown by the smooth lines in Fig. 4. The optimal number of subcomponents is $n_{\mathrm{s}}=8$. Their positions are marked by vertical dotted lines in the panels in Fig. 4. The corresponding $\chi_{\min }^{2}=1.21$ (for 

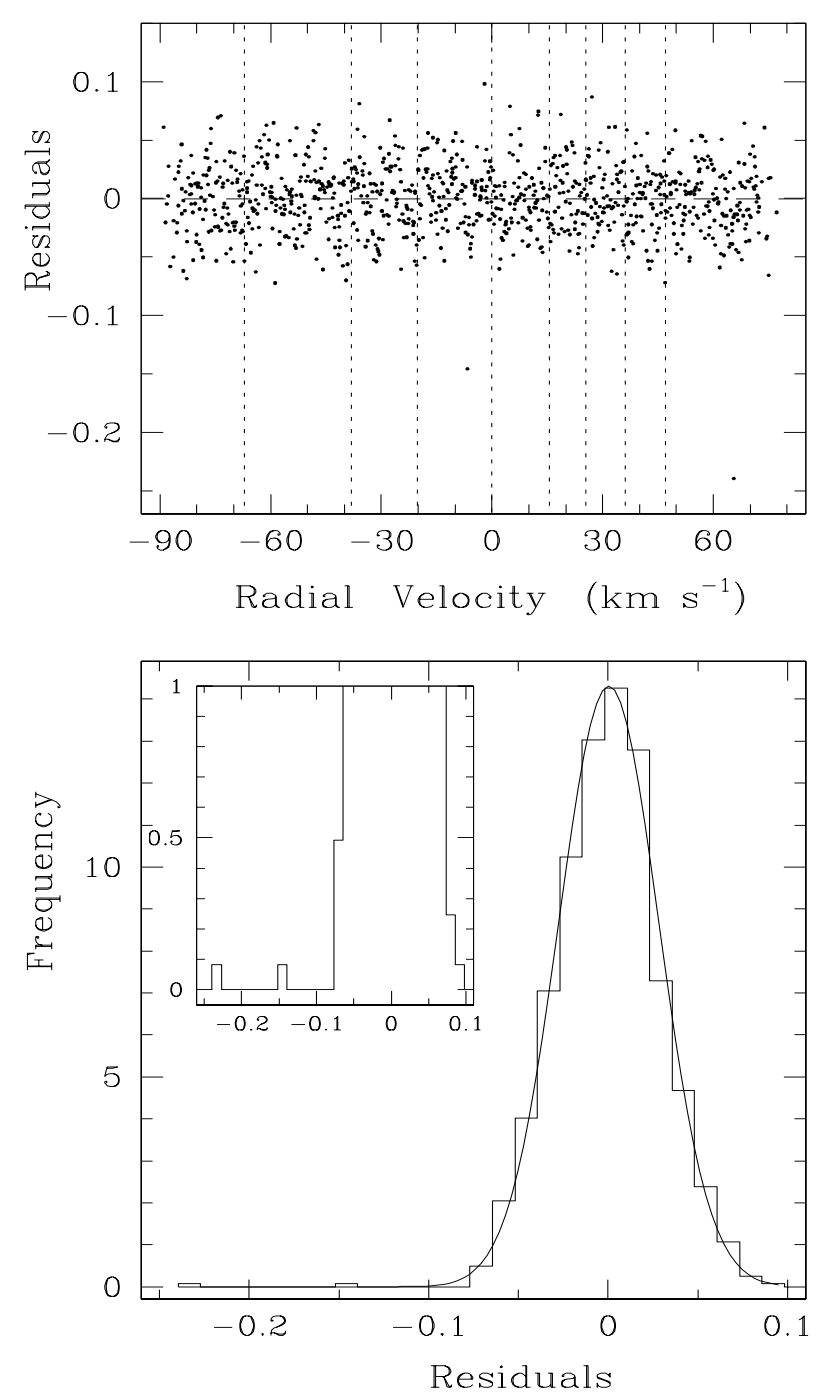

Fig. 5. Upper panel: the residuals $\epsilon=\left(\mathcal{F}^{\mathrm{cal}}-\mathcal{F}^{\mathrm{obs}}\right)$ for all Fe II profiles from Fig. 4. Dashed vertical lines indicate the positions of the subcomponents. Lower panel: shown are the distribution of $\epsilon$ (histogram) and the overplotted normal distribution (smooth curve) with the sample mean $\langle\epsilon\rangle=3.5 \times 10^{-4}$ and the dispersion $\sigma_{\epsilon}=2.9 \times 10^{-2}$ estimated from the total data set except for two "hot pixels" with $\epsilon<-0.1$ (seen in the inset).

$m=975$ and $p=39)$ is, however, too high: with $v=936$, the expected mean is $\chi_{v}^{2}=1 \pm 0.05(1 \sigma$ c.l.). However, the analysis of the residuals $\epsilon=\left(\mathcal{F}^{\mathrm{cal}}-\mathcal{F}^{\text {obs }}\right)$ shown in Fig. 5 reveals two "hot pixels" with $\epsilon<-0.1$ which deteriorate this $\chi_{\min }^{2}$ value $^{3}$. After removing these points, we find $\chi_{\min }^{2}=1.097$ (for $m=973$ and $p=39$ ).

The estimated wavelengths of the main Fe II components are listed in Table 3. These are the best fitting quantities. We do not calculate their errors since the spectral data from different exposures show very similar $S / N$ ratios which allow us to calculate the sample mean $\langle\Delta \alpha / \alpha\rangle$ without weights. The results are given in Col. 6 of Table 3. Column 7 lists the

\footnotetext{
3 The first "hot pixel" with $\epsilon=-0.15$ is seen in Fig. 4, exposures 23/24, panel Fe II 22586.6 , at $v=-66.6 \mathrm{~km} \mathrm{~s}^{-1}$, whereas the second one with $\epsilon=-0.24$, the same exposures, panel Fe II $\lambda 1608.5$, $v=65.6 \mathrm{~km} \mathrm{~s}^{-1}$ is beyond the frame.
}

Fe II pairs used in a particular $\Delta \alpha / \alpha$ measurement. We find $\langle\Delta \alpha / \alpha\rangle=(2.4 \pm 3.8) \times 10^{-6}$, and the corresponding root mean square $\sigma_{\mathrm{rms}}=1.3 \times 10^{-5}$.

\section{The fundamental noise limitation in the $\Delta \alpha / \alpha$ measurement}

In Sect. 4, we described the computational procedure used in this study - the single ion differential $\alpha$ measurement, SIDAM. A simple form of the deduced Eqs. (4) and (10) allows us to compute directly the fundamental uncertainty in the $\Delta \alpha / \alpha$ measurement due to photon noise. This analysis is based on the results obtained by Connes (1985) and by Bouchy et al. (2001) who calculated the fundamental noise limitation in the Doppler shift measurements.

Let us consider a digitalized and calibrated spectra of a pair of Fe II lines which are obtained with a high stability spectrograph. Let $\Delta \lambda_{\text {pix }}$ be the pixel size (the wavelength interval between pixels). Assume further that the spectrograph point-spread function can be described by a Gaussian with $F W H M_{\text {sp }}=2 \Delta \lambda_{\text {pix }}$ (the Nyquist limit). The observed Fe II lines are supposed to be isolated, single component, and unsaturated. Their apparent width, $F W H M_{\text {line }}$, is caused by the convolution of the spectrograph point-spread function with the "true" profile. The width of the true profile is defined by the quadratic sum of the thermal and turbulent components: $F W H M_{\text {true }}^{2}=$ $F W H M_{\text {therm }}^{2}+F W H M_{\text {turb }}^{2}$. Since Fe II lines are usually observed in the damped Ly- $\alpha$ systems where kinetic temperature is low $\left(\sim 100 \mathrm{~K}, F W H M_{\text {therm }} \sim 0.3 \mathrm{~km} \mathrm{~s}^{-1}\right)$ and the turbulent broadening is a few $\mathrm{km} \mathrm{s}^{-1}, F W H M_{\text {true }}$ might be less or about $F W H M_{\mathrm{sp}}$.

The error in the line center caused by counting statistics is given by Bohlin et al. (1983, Eq. (A15)):

$\sigma_{\lambda}=\frac{\Delta \lambda_{\text {pix }}}{W_{\text {obs }}} \frac{1}{\sqrt{\mathcal{N}_{\mathrm{e}}}} \frac{M \sqrt{M}}{\sqrt{12}} \Delta \lambda_{\text {pix }}$,

where $W_{\text {obs }}$ is the observed equivalent width, $\mathcal{N}_{\mathrm{e}}$ is the mean number of photoelectrons per pixel at the continuum level, and $M$ is the number of pixels covering the line profile ${ }^{4}$.

For Gaussian profiles, $M$ can be equal to $2.5 F W H M_{\text {line }} / \Delta \lambda_{\text {pix }}$, producing

$M=5 \xi$,

where $\xi=\left[1+\left(F W H M_{\text {true }} / F W H M_{\mathrm{sp}}\right)^{2}\right]^{1 / 2}$.

By analogy with the Connes procedure, we can characterize a line profile by a dimensionless quality factor $Q$ which is independent on the flux:

$Q=\frac{W_{\text {obs }}}{\Delta \lambda_{\text {pix }}} \frac{\sqrt{12}}{5 \sqrt{5} \xi \sqrt{\xi}}$,

and re-write Eq. (18) in the form

$\sigma_{\lambda}=\frac{\Delta \lambda_{\text {pix }}}{Q \sqrt{\mathcal{N}_{\mathrm{e}}}}$

${ }^{4}$ The second term in Bohlin's et al. Eq. (A15) is neglected since we assume the lines are rather strong (the apparent central optical depth $\tau_{0} \sim 1$ ), and isolated, i.e. the local continuum level is known with a sufficiently high precision. 
Table 3. SIDAM analysis: optimized centroid positions of the Fe II lines in the main subcomponent and $\Delta \alpha / \alpha$ calculated with Eqs. (4) or (10).

\begin{tabular}{|c|c|c|c|c|c|c|}
\hline $\begin{array}{l}\text { Exp. } \\
\text { No. } \\
(1)\end{array}$ & $\begin{array}{c}\lambda_{\mathrm{obs}}, \AA \\
\lambda 1608 \\
(2)\end{array}$ & $\begin{array}{c}\lambda_{\mathrm{obs}}, \AA \\
\lambda 2382 \\
(3)\end{array}$ & $\begin{array}{c}\lambda_{\mathrm{obs}}, \AA \\
\lambda 2586 \\
(4)\end{array}$ & $\begin{array}{c}\lambda_{\mathrm{obs}}, \AA \\
\lambda 2600 \\
(5)\end{array}$ & $\begin{array}{c}(\Delta \alpha / \alpha) \\
\text { (in units of } 10^{-5} \text { ) } \\
(6)\end{array}$ & $\begin{array}{c}\text { pair, } \\
\lambda_{1} / \lambda_{2} \\
\text { (7) }\end{array}$ \\
\hline \multirow[t]{3}{*}{$11 / 12$} & 4566.24408 & 6764.43913 & 7343.27915 & 7381.65008 & 1.199 & $1608 / 2382$ \\
\hline & & & & & -2.103 & $1608 / 2586$ \\
\hline & & & & & 0.098 & $1608 / 2600$ \\
\hline \multirow[t]{3}{*}{$19 / 20$} & 4566.23755 & 6764.44738 & 7343.26356 & 7381.64359 & -1.047 & $1608 / 2382$ \\
\hline & & & & & -1.525 & $1608 / 2586$ \\
\hline & & & & & -0.393 & $1608 / 2600$ \\
\hline \multirow[t]{3}{*}{$21 / 22$} & 4566.24661 & 6764.43913 & 7343.25994 & 7381.63961 & 1.668 & $1608 / 2382$ \\
\hline & & & & & 0.539 & $1608 / 2586$ \\
\hline & & & & & 1.860 & $1608 / 2600$ \\
\hline \multirow[t]{5}{*}{$23 / 24$} & 4566.25102 & 6764.44933 & 7343.27203 & 7381.65019 & 1.209 & $1608 / 2382$ \\
\hline & & & & & -0.028 & $1608 / 2586$ \\
\hline & & & & & 1.442 & $1608 / 2600$ \\
\hline & & & \multicolumn{2}{|c|}{ sample mean $\langle\Delta \alpha / \alpha\rangle$ : } & 0.24 & \\
\hline & & & \multicolumn{2}{|c|}{ error of the mean $\sigma / \sqrt{n}$ : } & 0.38 & \\
\hline
\end{tabular}

Table 4. Fundamental photon noise limits $\tilde{\sigma}_{\Delta \alpha / \alpha}^{\lim }$ (in units of $10^{-5}$ ) for Fe II pairs of lines $(\lambda 1608 / X)$ from the $z_{\text {abs }}=1.839$ system toward Q 1101-264. The exposure time $t_{\text {exp }}$ and the total efficiency $\varepsilon_{\text {tot }}$ are set to $3600 \mathrm{~s}$ and 0.15 , respectively.

\begin{tabular}{lllll}
\hline \hline \multicolumn{1}{r}{ Line: } & $\lambda 1608$ & $\lambda 2382$ & $\lambda 2586$ & $\lambda 2600$ \\
\hline$W_{\text {obs }}, \AA$ & 0.0446 & 0.2779 & 0.1239 & 0.2770 \\
$\Delta \lambda_{\text {pix }}, \AA$ & 0.05 & 0.06 & 0.05 & 0.05 \\
$\xi$ & 1.83 & 1.83 & 1.93 & 1.93 \\
$Q$-factor & 0.11 & 0.58 & 0.29 & 0.64 \\
$\sigma_{\lambda}^{\lim }, \mathrm{m} \AA$ & 5.7 & 1.3 & 2.3 & 1.0 \\
$\tilde{\sigma}_{\Delta \alpha / \alpha}^{\lim }$ & - & 1.1 & 1.0 & 1.1 \\
\hline
\end{tabular}

This equation shows that the error $\sigma_{\lambda}$ decreases almost quadratically with decreasing wavelength bin per pixel ( $W_{\text {obs }}$ does not depend on the spectral resolution, and $\xi \sim \sqrt{2}$, if $F W H M_{\mathrm{sp}} \sim$ $\left.F W H M_{\text {true }}\right)$ under the assumption that $N_{\mathrm{e}}$ is keeping constant. The quality factor for Fe II lines from the $z_{\mathrm{abs}}=1.839$ system ranges between 0.1 and 0.7 (Table 4 ).

The total number of photoelectrons can be estimated from the specific flux $J_{v}^{V}=3.81 \times 10^{-20} \mathrm{ergs} \mathrm{s}^{-1} \mathrm{~cm}^{-2} \mathrm{~Hz}^{-1}$ of a $m v=0$ star outside the Earth's atmosphere $: F_{*}^{V}=1.05 \times$ $10^{3}$ photon $\mathrm{cm}^{-2} \mathrm{~s}^{-1} \AA^{-1}$. Then $\mathcal{N}_{\mathrm{e}}$ is given by:

$\mathcal{N}_{\mathrm{e}}=\frac{F_{*}^{V} s_{\text {tel }} \varepsilon_{\text {tot }} t_{\text {exp }} \Delta \lambda_{\text {pix }}}{10^{0.4 m v}}$

Here $s_{\text {tel }}$ is the telescope area in $\mathrm{cm}^{2} ; \varepsilon_{\text {tot }}$ the overall detection efficiency of the telescope, spectrograph and detector, corrected for the contribution of the atmosphere; $t_{\exp }$ the exposure time in $\mathrm{s} ; m v$ the visual magnitude of the quasar.

At $5500 \AA$, the UVES efficiency $\varepsilon_{\text {tot }} \simeq 0.15$ (Kaufer et al. 2004), and with a $3600 \mathrm{~s}$ exposure $\left(\mathcal{N}_{\mathrm{e}} \simeq 5670\right)$, one

\footnotetext{
${ }^{5}$ For $B$ and $R$ filters, the corresponding intensities are $F_{*}^{B}=1.52 \times$ $10^{3}$ and $F_{*}^{R}=0.65 \times 10^{3}$ photon $\mathrm{cm}^{-2} \mathrm{~s}^{-1} \AA^{-1}$.
}

expects a fundamental uncertainty of about 1-5 mA in $\sigma_{\lambda}$ for a $m v=16 \mathrm{QSO}$. Then, (11) provides, respectively, the fundamental noise of $\sim(0.2-1.0) \times 10^{-5}$ in $\tilde{\sigma}_{\Delta \alpha / \alpha}$ for one measurement of a Fe II pair of lines.

The fundamental photon noise limits for Fe II pairs from the $z_{\mathrm{abs}}=1.839$ system are given in Table 4 . The photon noise is computed with the best-fitting parameters $N_{1}=1.335 \times$ $10^{13} \mathrm{~cm}^{-2}$ and $b_{1}=5.25 \mathrm{~km} \mathrm{~s}^{-1}$.

\section{Discussion and outlook}

\section{1. $\Delta \alpha / \alpha$ from different studies}

Measurements from the present paper can be combined with the previous Fe II sample from the $z_{\mathrm{abs}}=1.15$ system toward HE 0515-4414 (QRL) to increase statistics. The normalized distribution $\left(\int \cdot \mathrm{d} \Delta \alpha / \alpha=1\right)$ of the resulting $35 \Delta \alpha / \alpha$ values is plotted in Fig. 6 (histogram) along with two other recently published results of MFWDPW and CSPA which are shown by the dashed and dotted curves, respectively, assuming that the measured $\Delta \alpha / \alpha$ are normally distributed with the sample means and standard deviations published in these papers. The vertical lines in this figure mark the centers of the corresponding distributions. The distribution shown by the histogram has the sample mean $\langle\Delta \alpha / \alpha\rangle=\left(0.4 \pm 1.5_{\text {stat }}\right) \times 10^{-6}$ and the median $(\Delta \alpha / \alpha)_{\text {med }}=0.1 \times 10^{-6}$.

It is to be noted that at a given redshift the sample mean $\langle\Delta \alpha / \alpha\rangle$ should be the same within the uncertainty interval independently on the method or the sample used - provided the data are free from any systematics. The results presented in Fig. 6 show, however, that $\langle\Delta \alpha / \alpha\rangle_{\text {Keck/HIRES }} \neq\langle\Delta \alpha / \alpha\rangle_{\mathrm{VLT} / \mathrm{UVES}}$. The sample means of CSPA and our Fe II ensemble are in good agreement but they differ from that of MFWDPW at the 


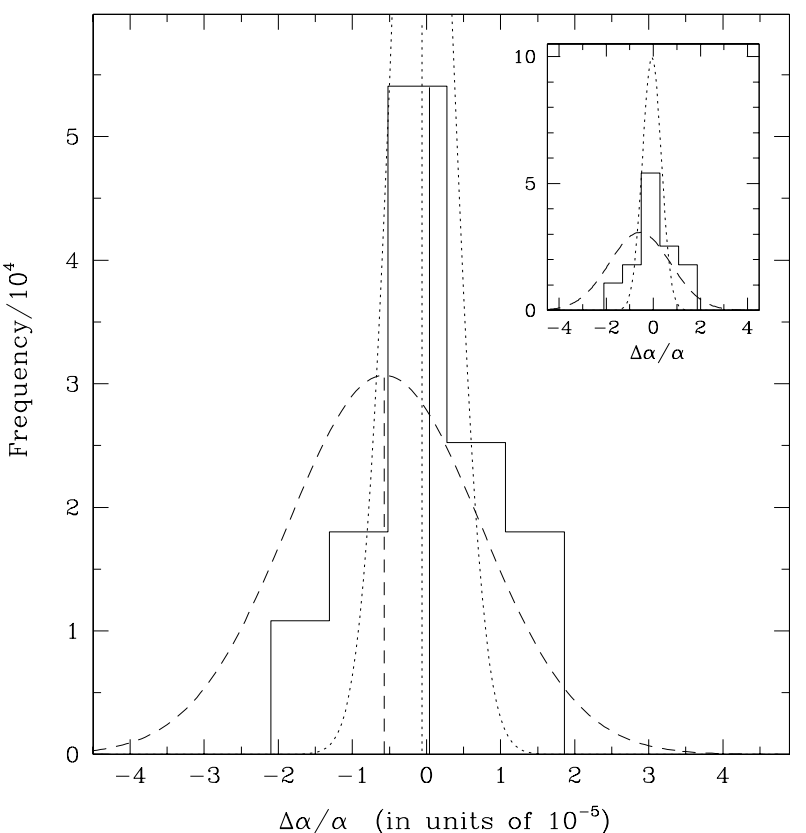

Fig. 6. The distribution of $\Delta \alpha / \alpha$ (histogram) from the $z_{\mathrm{abs}}=1.839$ and $z_{\text {abs }}=1.15$ Fe II systems toward Q 1101-264 and HE 0515-4414, respectively. The distributions of $\Delta \alpha / \alpha$ from MFWDPW (dashed curve) and CSPA (dotted curve) are overplotted to demonstrate the discrepancy between the Keck/HIRES and VLT/UVES sample mean $\langle\Delta \alpha / \alpha\rangle$ values. Both MFWDPW and CSPA distributions are assumed to be normal with the means and variances taken from the corresponding papers. The vertical lines mark the centers of the distributions. The revealed Keck-VLT discrepancy of $\langle\Delta \alpha / \alpha\rangle$ is statistically significant at the $95 \%$ confidence level according to the $t$-test.

95\% significance level according to the $t$-test ${ }^{6}$. This discrepancy points to the systematic shift which mimics the effect of varying $\alpha$ in the Keck/HIRES spectra. To clarify the origin of this systematic shift, one needs more accurate measurements, which can be carried out with higher spectral resolution and with more homogeneous samples.

The comparison of the distribution widths in Fig. 6 reveals that the standard deviation in the CSPA sample is exceptionally small. For example, Fig. 1b in CSPA, where the accuracy of wavelength calibration is checked through the relative velocity shifts, $\Delta v$, between the Fe II $\lambda 2344$ and $\lambda 2600$ lines $^{7}$, shows the dispersion of $\sigma_{\Delta v} \simeq 0.4 \mathrm{~km} \mathrm{~s}^{-1}$. This uncertainty in wavelength calibration transforms into the error $\sigma_{\Delta \alpha / \alpha} \sim 2 \times 10^{-5}$ (see Eq. (12) in L04), i.e., in order to reach the error of the mean $\sigma_{\langle\Delta \alpha / \alpha\rangle} \sim 0.6 \times 10^{-6}$ (CSPA), one needs a sample of the size $n \sim 1100$, which is not the case. Thus, the error of the mean $\sigma_{\langle\Delta \alpha / \alpha\rangle}$ estimated by CSPA is in some disagreement with their Fig. 1b. The scatter of $\Delta \alpha / \alpha$ in the Keck sample is about 2 times the $\sigma_{\text {rms }}$ value of our combined Fe II sample.

${ }^{6}$ The application of the $t$-test to two normally distributed and independent random variables with different variances is described in Bol'shev \& Smirnov (1983).

7 The mean $\langle\Delta v\rangle$ should be consistent with zero in the case of good calibration since the sensitivity coefficients $Q(\mathrm{Fe}$ II $\lambda 2344) \simeq$ $Q(\mathrm{Fe}$ II $\lambda 2600)$.

\subsection{Future prospects}

Future measurements of $\Delta \alpha / \alpha$ from astronomical observations should be carried out with high precision to check the present Oklo result that $\alpha$ was larger in the past, $\Delta \alpha / \alpha \geq 4.5 \times 10^{-8}$ (Lamoreaux \& Torgerson 2004). To reach the Oklo scale of $\alpha$-variation, one needs the accuracy of at least $\sigma_{\Delta \alpha / \alpha} \sim 10^{-7}$, which is 8 times higher than the accuracy set by the errors in the laboratory wavelengths (Sect. 4.1). At first glance the Oklo level is unachievable in astronomical observations. However, applying the SIDAM method to a few Fe II systems observed at different redshifts, one can omit the laboratory wavelengths from $\Delta \alpha / \alpha$ calculations. Such self-calibrating procedure, described by BSS, implies that the measured values of $R(t)$, Eq. (7), can be fitted to a linear function of cosmic time given in the form (cf. Eq. (6) in BSS):

$R(t)=R(0)\left(1+S t H_{0}\right)$,

where $R(0)$ is any convenient constant, $H_{0}$ is the Hubble constant, and the slope $S$ is equal in our case to

$S=\frac{\kappa}{H_{0} \alpha}\left(\frac{\mathrm{d} \alpha}{\mathrm{d} t}\right)$

The constant $\kappa$ is defined through Eq. (10): $\kappa=4\left(Q_{1}-Q_{2}\right)(\eta+$ $1) / \eta(\eta+2)$. For the pair Fe II $\lambda \lambda 1608,2586, \kappa \simeq-1 / 4$.

Equation (21) shows that for narrow lines $(\xi<\sqrt{2})$ the error $\sigma_{\lambda}$ decreases almost quadratically with decreasing wavelength bin per pixel if $N_{\mathrm{e}}$ is fixed. Therefore, if an efficient spectrograph with ten times the UVES dispersion and superior stability can be coupled to a $100 \mathrm{~m}$ class telescope, one would expect $\sigma_{\lambda}^{\lim } \sim 0.03-0.05 \mathrm{~m} \AA$, and, thus, the precision of $\sim 10^{-7}$ in the $\Delta \alpha / \alpha$ measurements can be achieved. Then, with good statistics the Oklo result can be checked at different redshifts.

If $\alpha$ were indeed larger in the past, then the logarithmic derivative of $\alpha(t)$ in Eq. (24) is negative (since $t$ decreases with increasing redshift) and we would observe a positive slope $S$. Otherwise, if $\alpha$ were smaller in the past, $S$ would be negative.

\section{Conclusions}

The main results of the present paper are as follows:

1. A data reduction procedure is designed to control the accuracy of the wavelength scale calibration and to bypass the influence of the spectral shifts due to instabilities in the instrument which perturb the narrow absorption-line profiles.

2. A single ion method is proposed to minimize the influence of the systematic shifts of the line centroids to the accuracy of the $\Delta \alpha / \alpha$ measurements.

3. The mean value $\langle\Delta \alpha / \alpha\rangle=\left(2.4 \pm 3.8_{\text {stat }}\right) \times 10^{-6}$ is obtained from the analysis of the $z_{\mathrm{abs}}=1.839$ Fe II system toward Q 1101-264. Combination of this measurement with the $z_{\mathrm{abs}}=1.15$ Fe II system toward HE 0515-4414 (QRL) increases the sample size to $35 \mathrm{Fe}$ II pairs and provides $\langle\Delta \alpha / \alpha\rangle=\left(0.4 \pm 1.5_{\text {stat }}\right) \times 10^{-6}$.

4. We confirm that the VLT/UVES estimations of $\Delta \alpha / \alpha$ differ significantly from the Keck/HIRES result, and that the systematic shift is present in the Keck data with a probability of $95 \%$. 
5. The fundamental noise limitation in the $\Delta \alpha / \alpha$ measurement is defined and calculated for the $z_{\text {abs }}=1.839$ Fe II system. It is shown that a typical VLT/UVES fundamental noise limit is $\sigma_{\Delta \alpha / \alpha}^{\lim } \sim 1 \times 10^{-5}$ for a pair of Fe II lines observed in a spectrum of a relatively bright QSO $(V=$ 16.0). This estimation is robust since the inclusion of other metal transitions (like Mg II $\lambda \lambda 2796,2803$; Al II $\lambda 1670$; Si II $\lambda \lambda 1526,1808)$ cannot improve significantly the error $\sigma_{\Delta \alpha / \alpha}^{\lim }$ due to lower sensitivity to the variation of $\alpha$. From this point of view the dispersion $\sigma_{\Delta \alpha / \alpha}=0.4 \times 10^{-5}$ and the error of the mean $\sigma_{\langle\Delta \alpha / \alpha\rangle}=0.6 \times 10^{-6}$ found in CSPA seems to be several times underestimated.

6. We suggest that with a spectrograph of $\sim 10$ times the UVES dispersion and superior stability the accuracy high enough to probe the Oklo result, $\Delta \alpha / \alpha \geq 4.5 \times 10^{-8}$, can be attained in future at new giant telescopes.

Acknowledgements. The authors are indebted to Ralf Quast for his valuable comments. S.A.L. gratefully acknowledges the hospitality of Osservatorio Astronomico di Trieste where this work was performed under the program COFIN 02 N 2002027319-001. The work of S.A.L. is supported by the RFBR grant No. 03-02-17522 and by the RLSS grant 1115.2003.2.

\section{References}

Ashenfelter, T., Mathews, G. J., \& Olive, K. A. 2004, Phys. Rev. Lett., 92, 041102

Bahcall, J. N., Sargent, W. L. W., \& Schmidt, M. 1967, ApJ, 149, L11

Bahcall, J. N., Steinhardt, C. L., \& Schlegel, D. 2004, ApJ, 600, 520 (BSS)

Bohlin, R. C., Hill, J. K., Jenkins, E. B., et al. 1983, ApJS, 51, 277

Boksenberg, A., \& Snijders, M. A. J. 1981, MNRAS, 194, 353

Bol'shev, L. N., \& Smirnov, N. B. 1983, Tables of Mathematical Statistics (Moscow: Nauka)

Bouchy, F., Pepe, F., \& Queloz, D. 2001, A\&A, 374, 733

Carswell, R. F., Whelan, J. A. J., Smith, M. G., Boksenberg, A., \& Tytler, D. 1982, MNRAS, 198, 91

Carswell, R. F., Morton, D. C., Smith, M. G., et al. 1984, ApJ, 278, 486

Chand, H., Srianand, R., Petitjean, P., \& Aracil, B. 2004, A\&A, 417, 853 [CSPA]

Chieffi, A., \& Limongi, M. 2004, ApJ, 608, 405

Connes, P. 1985, Ap\&SS, 110, 211

Dirac, P. A. M. 1937, Nature, 139, 323

Dessauges-Zavadsky, M., Péroux, C., Kim, T.-S., D’Odorico, S., \& McMahon, R. G. 2003, MNRAS, 345, 447

Drullinger, R. E., Wineland, D. J., \& Bergquist, J. C. 1980, Appl. Phys., 22, 365
Dzuba, V. A., Flambaum, V. V., \& Webb, J. K. 1999, Phys. Rev. A, 59, 230

Dzuba, V. A., Flambaum, V. V., Kozlov, M. G., \& Marchenko, M. V. 2002, Phys. Rev. A, 66, 022501

Edlén, B. 1966, Metrologia, 2, 71

Fischer, M., Kolachevsky, N., Zimmermann, M., et al. 2004, Phys. Rev. Lett., 92, 230802

Kaufer, A., D'Odorico, S., \& Kaper, L. 2004, UV-Visual Echelle Spectrograph. User Manual (http://www.eso.org/ instruments/uves/userman/), 40

Kim, T.-S., Cristiani, S., \& D'Odorico, S. 2002, A\&A, 383, 747

Kozlov, M. G., Korol, V. A., Berengut, J. C., Dzuba, V. A., \& Flambaum, V. V. 2004, Phys. Rev. A, 70, 062108 (KKBDF)

Lamoreaux, S. K., \& Torgerson, J. R. 2004, Phys. Rev. D, 69, 121701

Lanzetta, K. M., Wolfe, A. M., \& Turnshek, D. A. 1987, ApJ, 322, 739

Levshakov, S. A. 2004, in Astrophysics, Clocks and Fundamental Constants, ed. S. G. Karshenboim, \& E. Peik (Berlin, Heidelberg: Springer-Verlag), 151 (L04)

Levshakov, S. A., Dessauges-Zavadsky, M., D’Odorico, S., \& Molaro, P. 2002a, ApJ, 565, 696

Levshakov, S. A., Dessauges-Zavadsky, M., D’Odorico, S., \& Molaro, P. 2002b, MNRAS, 333, 373

Levshakov, S. A. 1994, MNRAS, 269, 339

Marciano, W. J. 1984, Phys. Rev. Lett., 52, 489

Milne, E. A. 1937, Proc. R. Soc. A, 158, 324

Mohr, P. J., \& Tailor, B. N. 2000, Rev. Mod. Phys., 72, 351

Morton, D. C. 2003, ApJS, 149, 205

Mota, D. F., \& Barrow, J. D. 2004, MNRAS, 349, 291

Murphy, M. T., Webb, J. K., Flambaum, V. V., Prochaska, J. X., \& Wolfe, A. M. 2001, MNRAS, 327, 1237

Murphy, M. T., Webb, J. K., \& Flambaum, V. V. 2003, MNRAS, 345, 609

Murphy, M. T., Flambaum, V. V., Webb, J. K., et al. 2004, in Astrophysics, Clocks and Fundamental Constants, ed. S. G. Karshenboim, \& E. Peik (Berlin, Heidelberg: Springer-Verlag), 131 (MFWDPW)

Olive, K. A., Pospelov, M., Qian, Y.-Z., et al. 2004, Phys. Rev. D, 69, 027701

Osmer, P. S., \& Smith, M. G. 1977, ApJ, 213, 607

Pepe, F., Mayor, M., Queloz, D., et al. 2004, A\&A, 423, 385

Petitjean, P., Srianand, R., \& Ledoux, C. 2000, A\&A, 364, L26

Potekhin, A. Y., Ivanchik, A. V., Varshalovich, D. A., et al. 1998, ApJ, 505, 523

Quast, R., Reimers, D., \& Levshakov, S. A. 2004, A\&A, 415, L7 (QRL)

Savedoff, M. P. 1956, Nature, 178, 688

Uzan, J.-P. 2003, Rev. Mod. Phys., 75, 403

Varshalovich, D. A., \& Levshakov, S. A. 1993, JETP Lett., 58, 237

Webb, J. K., Flambaum, V. V., Churchill, C. W., Drinkwater, M. J., \& Barrow, J. D. 1999, Phys. Rev. Lett., 82, 884

Young, P., Sargent, W. L. W., \& Boksenberg, A. 1982, ApJS, 48, 455 